\title{
Itinéraires Itinéraires
}

Littérature, textes, cultures

2019-2 et $3 \mid 2019$

Corps masculins et nation : textes, images, représentations

\section{Un cinéma viril pour une Espagne virile : le combat de Nuestro Cinema (1932-1935)}

A Virile Cinema for a Virile Spain: the Struggle of Nuestro Cinema (1932-1935)

Évelyne Coutel

(2) OpenEdition

Journals

Édition électronique

URL : http://journals.openedition.org/itineraires/6698

DOI : $10.4000 /$ itineraires.6698

ISSN : 2427-920X

Éditeur

Pléiade

Référence électronique

Évelyne Coutel, « Un cinéma viril pour une Espagne virile : le combat de Nuestro Cinema (1932-1935) 》, Itinéraires [En ligne], 2019-2 et 3 | 2019, mis en ligne le 13 décembre 2019, consulté le 15 décembre 2019. URL : http://journals.openedition.org/itineraires/6698; DOI : 10.4000/itineraires.6698

Ce document a été généré automatiquement le 15 décembre 2019.

\section{cc) $(1) \ominus$}

Itinéraires est mis à disposition selon les termes de la licence Creative Commons Attribution - Pas d'Utilisation Commerciale - Pas de Modification 4.0 International. 


\section{Un cinéma viril pour une Espagne virile : le combat de Nuestro Cinema (1932-1935)}

A Virile Cinema for a Virile Spain: the Struggle of Nuestro Cinema (1932-1935)

\section{Évelyne Coutel}

1 Les premières revues de cinématographie ayant vu le jour dès les années 1910 correspondaient, dans leur majorité, à un modèle de presse dite " populaire » en raison de son caractère hétéroclite qui la destinait au grand public ${ }^{1}$. Parmi les principaux ingrédients qui alimentent ces premières revues et qui expliquent leur popularité auprès des lecteurs, il convient de citer le star system, qui est à son tour fomenté et consolidé par la presse de cinéma à travers la publication de photographies, d'interviews ou même de critiques de films qui, à cette époque, se centrent volontiers sur le jeu des interprètes. Il faut souligner d'emblée que la présence du star system au sein de ces publications leur confère une certaine "féminité » qui se manifeste en premier lieu dans les couvertures qui, la plupart du temps, font apparaître le visage d'une actrice issue du cinéma hollywoodien ou national. Celle-ci est parfois accompagnée d'un partenaire filmique, jeune premier ou acteur confirmé dont on ne peut que constater la prestance et l'élégance. Ce type de couverture dirige l'attention vers la thématique centrale de nombreux films : l'amour et les relations sentimentales.

2 Parallèlement à ce modèle de revue populaire se développe, dès les années 1930 voire la fin des années 1920, une presse à caractère savant et professionnalisant, qui entend faire le tri entre de «bons » et de «mauvais » usages du cinéma et qui hiérarchise les productions filmiques en fonction de critères bien précis, notamment de leur rattachement éventuel au star system qui constitue un facteur de dénigrement. La revue qui incarne sans doute le mieux cette alternative est Nuestro Cinema, fondée et dirigée par Juan Piqueras, « le Delluc espagnol ", d'après l'expression de Sadoul ${ }^{2}$ (Llopis 1988). Cette revue se présente constamment comme la «meilleure », celle qui se distingue de la plupart des autres par un contenu plus « sérieux » et par la considération du cinéma en tant qu'art ${ }^{3}$, ce qui passe par une incitation permanente à le « purger » des éléments 
qui le réduisent à un simple divertissement. Le mépris exprimé par Piqueras à l'égard des autres revues va de pair avec celui de la production cinématographique nationale. Piqueras estime qu'en l'état actuel le cinéma espagnol n'est qu'une industrie, un loisir sans transcendance: "à cet instant même où nous évoquons le cinéma hispanique, nous devons nous en tenir à sa facette industrielle, étant donné que nous ne pouvons pas le considérer sous un autre angle ${ }^{4} »$ (Piqueras 1935). Concernant la presse spécialisée, il affirmera que "la presse cinématographique, en général, porte à son comble la vulgarité et la putréfaction parmi l'ensemble des publications actuelles ${ }^{5}$ ” (Piqueras 1933).

3 À partir de l'analyse des articles parus dans Nuestro Cinema, il s'agira de montrer que la dépréciation d'une culture cinématographique présentée comme triviale et antiartistique traduit en réalité une volonté de masculinisation de cette culture et de la cinéphilie. Ce phénomène se déploie dans un contexte spécifique. D’une part, au début $\mathrm{du} \mathrm{xx}^{\mathrm{e}}$ siècle, le cinéma fait progressivement l'objet d'une entreprise de légitimation artistique qui entraîne le rejet ou la mise à distance de ses composantes " féminines ", conformément à un schéma qui consiste à associer l'art et la création artistique ou littéraire au genre masculin (Coquillat 1982). En outre, les évolutions et les nouveaux droits dont bénéficient les Espagnoles sous la Seconde République entraînent chez les hommes une crise identitaire (Aresti Esteban 2010) qui s'exprime dans la culture cinématographique. Comme l'a remarqué Patrick Farges (2012) pour le cas allemand, " la conquête par les femmes de nouveaux territoires (au sens d'espaces physiques autant que d'espaces symboliques) a suscité [...] diverses réactions de défense du territoire par des hommes habitués jusque-là à ne pas avoir de concurrence féminine ". En Espagne, il est logique qu'une telle contre-offensive ait lieu dans les années 1930, suite aux avancées inédites que la condition féminine connaît à cette période l'obtention du droit de vote en 1931 constitue le point culminant de ces changements et déclenche des ripostes antiféministes. Nuestro Cinema semble bien illustrer ces mécanismes: la culture cinématographique dont elle fait sa bête noire comporte un ensemble de productions et de pratiques perçues comme potentiellement dangereuses pour les hommes. Il est question, en effet, d'une culture accessible aux femmes, et qui semble même leur être particulièrement adressée en raison de l'orientation sentimentale de nombreux films. La masculinisation de la culture cinématographique qui s'opère dans les pages de Nuestro Cinema a pour but de promouvoir un cinéma «viril» et une conception de la nation fondée sur la domination masculine et sur le rejet de l'homosexualité ${ }^{\text {. }}$

\section{Une iconographie de la virilité}

4 Tout au long de sa parution, Nuestro Cinema n'aura de cesse de mettre à l'honneur une iconographie de la virilité en publiant des images qui exaltent la force physique, l'esprit de combat et la bravoure. Nul doute que cette iconographie s'inscrit dans un système patriarcal et qu'elle contribue à la défense d'une masculinité "hégémonique » et normative qui inclut une série de critères définissant la manière d'être un homme et qui « permet de légitimer d'un point de vue idéologique la subordination des femmes à l'égard des hommes » (Connell et Messerschmidt $2015: 155)$.

5 L'une des lignes directrices de la revue tient à l'éloge du cinéma russe et soviétique, et de ses cinéastes. Toutes les productions filmiques sont ainsi jugées par rapport à la 
norme du réalisme socialiste de l'URSS stalinienne. Cette tendance doit être mise en rapport, dans un premier temps, avec les tensions politiques qui agitent l'Espagne à ce moment et qui sont liées à la montée de la lutte des classes. Piqueras et les siens louent ce cinéma car, contrairement au cinéma hollywoodien - qualifié de cinéma «bourgeois ", au service des intérêts des classes les plus aisées et dépourvu de contenu social -, il prend en compte la situation du prolétariat en représentant des foules plutôt qu'une star - un seul individu - qui sera au centre du dispositif visuel et narratif de films visant à la promouvoir.

6 Si ces motivations sont primordiales et s'inscrivent logiquement dans l'engagement politique des journalistes qui écrivent dans Nuestro Cinema - lesquels ont alors adhéré au Parti communiste ${ }^{7}-$, il ne faut pas pour autant omettre d'autres motivations moins affichées, peut-être moins conscientes ou moins avouables.

7 Les ambitions plus profondes que revendique Nuestro Cinema face à l'ensemble de la presse cinématographique reposent en grande partie sur le rejet du star system qui constitue pour ces journalistes un important facteur de différenciation. Ce parti pris s'exprime dès les couvertures, nettement différentes de celles des revues « hybrides »: on peut y contempler des visages ou des corps d'hommes bien développés, entraînés au combat et à l'effort physique (Fig. 1 et 2). Des hommes au travail ou à la guerre, qui s'opposent radicalement à l'«oisiveté » des acteurs de films hollywoodiens dont l'énergie ne sert qu'à séduire une jeune femme ou à la reconquérir - entreprise guère valorisante, peu virile et bien dérisoire au regard des actions menées par les héros des films russes. Ces derniers ont d'ailleurs des caractéristiques physiques rassurantes, qui s'opposent à l'apparence physique de certaines stars masculines ayant suscité de fortes inquiétudes chez les journalistes espagnols.

Fig. 1. Couverture du numéro 5 de Nuestro Cinema (octobre 1932).

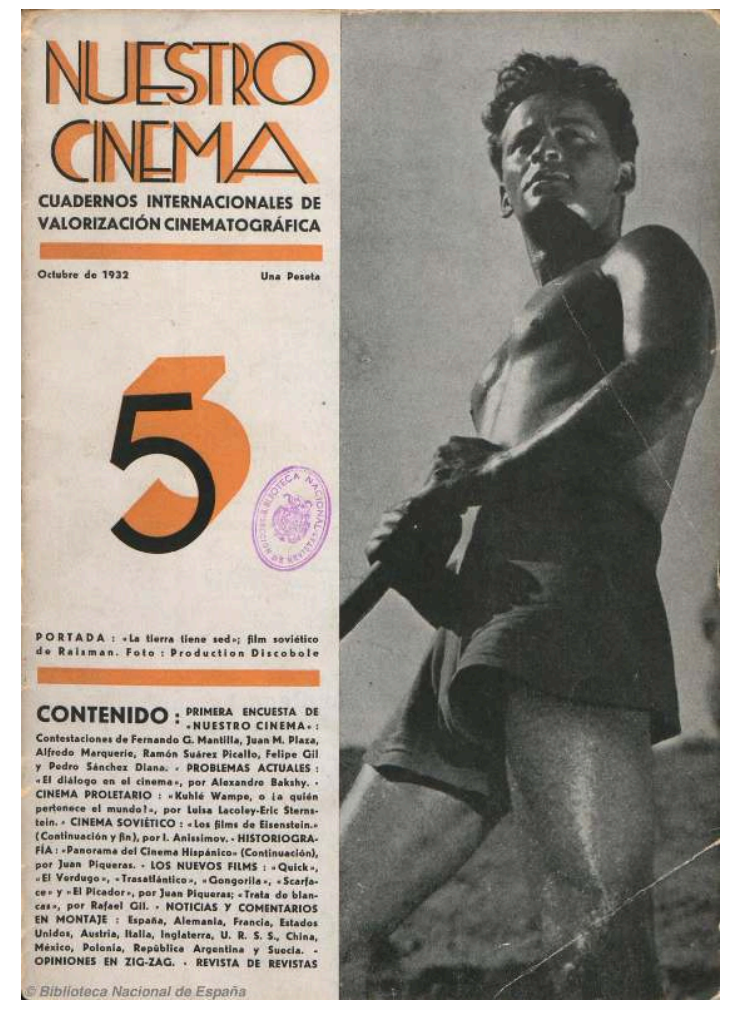

Photogramme extrait du film soviétique La Terre a soif(Youli Raizman, 1932). 
Fig. 2. Couverture du numéro 11 de Nuestro Cinema (avril-mai 1933).

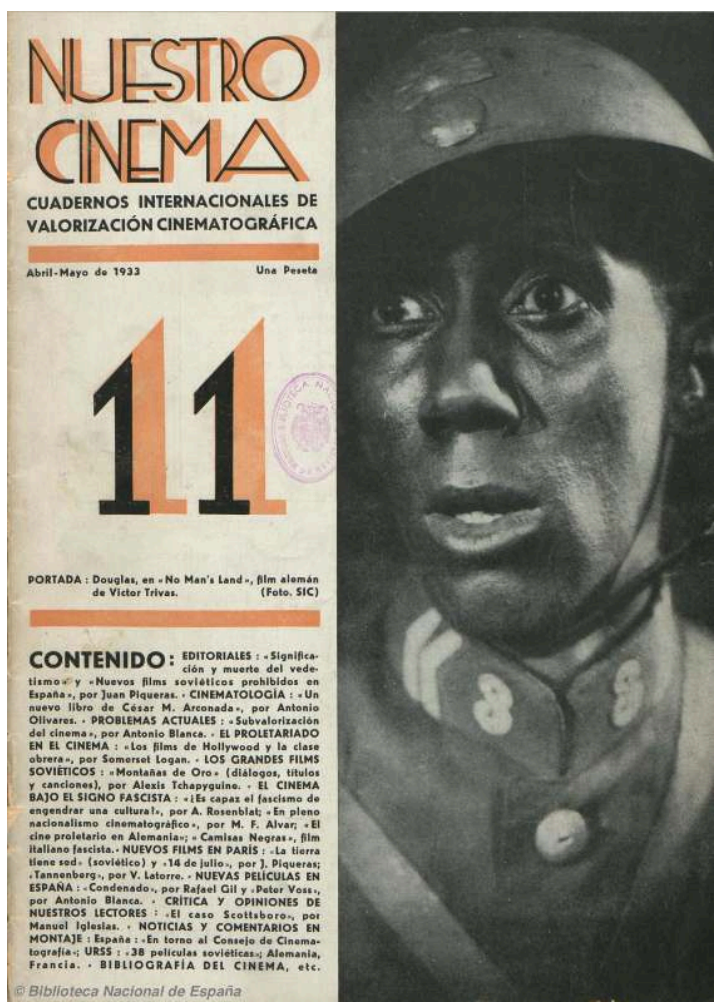

Photogramme extrait du film allemand No Man's Land (Victor Trivas, 1931).

Ces acteurs, qui ont marqué le cinéma hollywoodien des années 1920 et 1930, font l'objet de railleries dans la mesure où ils incarnent, aux yeux de certains spectateurs et journalistes, une masculinité dégradée et une virilité déchue. Parmi les éléments problématiques, il faut mentionner le mot anglais «star » qui est de genre féminin, ce que sa traduction en espagnol par «estrella » met d'autant plus en relief en raison du phonème [a]. L'application de ce mot à des acteurs de sexe masculin a donc pu être perçue comme problématique, comme l'atteste un éditorial de Cine Popular. Le directeur de cette revue, un dénommé "Aurelio », estime que le substantif «estrella» est tout à fait approprié dans le cas des actrices mais il s'insurge face à l'emploi du même terme pour les acteurs. Le corps de l'homme, moins sujet à un idéal de perfection, ne se prête pas, selon lui, à un tel usage. Mais ce qui rend le terme "estrella » inutilisable pour désigner les acteurs, c'est avant tout la désinence de genre liée au phonème [a] qui a un effet féminisant :

Les Anglais ne font pas de distinction de sexe entre les êtres inanimés et les choses d'une autre nature, mais en Espagne nous sommes plus judicieux et nous opérons une sage distinction, voire une séparation de sexe, dans la grammaire.

[...]

Nous n'acceptons pas que Charles Chaplin soit une "estrella", tout comme le fantastique et fougueux Douglas Fairbanks. [...]

Nous croyons qu'il serait plus altruiste de leur donner l'heureux appellatif de " luceros $^{8}$ ", ce qui instaurerait une sage différence entre « elles » et « eux », et ôterait à ces derniers ces jupes grammaticales que l'irréligiosité et l'iconoclastie de quelques hérétiques leur ont plaquées dans le plus grand ridicule.

Appelons-les "luceros", aussi resplendissants que leur vanité et leur valeur l'exigeront, mais laissons-leur, à « elles ", le qualificatif doucement féminin de " 
estrella », preuve ô combien éloquente, aimable et galante de notre reconnaissance envers ces belles et sympathiques femmes qui nous font passer de si agréables moments grâce à leurs grandes créations ${ }^{9}$. (Aurelio 1922)

L'idéal de perfection, de raffinement et d'élégance associé au terme " estrella » est jugé incompatible avec la virilité, une qualité que semblent avoir perdue certains acteurs qui seront stigmatisés par des journalistes qui, comme Piqueras, restent attachés à un idéal de masculinité fondé sur la virilité et la domination. Un poème satirique publié dans la revue Cinòpolis ${ }^{10}$ illustre bien les boutades suscitées par les acteurs qui, comme Adolphe Menjou, incarnent l'homme-objet, victime de son pouvoir d'attraction sur le public féminin : «Le smoking moiré / et le gilet en "tricot" / transforment, comme on peut le voir, / un doyen en bibelot ${ }^{11} »$ (Bishop 1929). L'élégance et la mode vestimentaire associées au costume de Menjou semblent donc avoir un effet dévirilisant. Un dessin représente l'acteur complètement nu, cachant son sexe avec ses mains (Fig. 3), tandis qu'un groupe d'admiratrices le poursuit. S'agissant du même acteur, le célèbre texte de Buñuel (1928) intitulé «Variations sur la moustache de Menjou » («Variaciones sobre el bigote de Menjou ») attire d'emblée l'attention sur la fine moustache qu'arborent de nombreux acteurs hollywoodiens et qui peut expliquer à elle seule la déconsidération de la culture cinématographique qui leur est associée. Cet attribut qui a remplacé la barbe s'oppose radicalement à l'hirsutisme ou à la moustache plus fournie qui, en revanche, se veulent récurrentes dans l'iconographie du réalisme socialiste, récupérée par la revue (Fig. 4 et 5).

Fig. 3. Poèmes et dessins satiriques d'Adolphe Menjou, par « Magnessie Bishop », Cinòpolis, 26 janvier 1929, $n^{\circ} 1$.

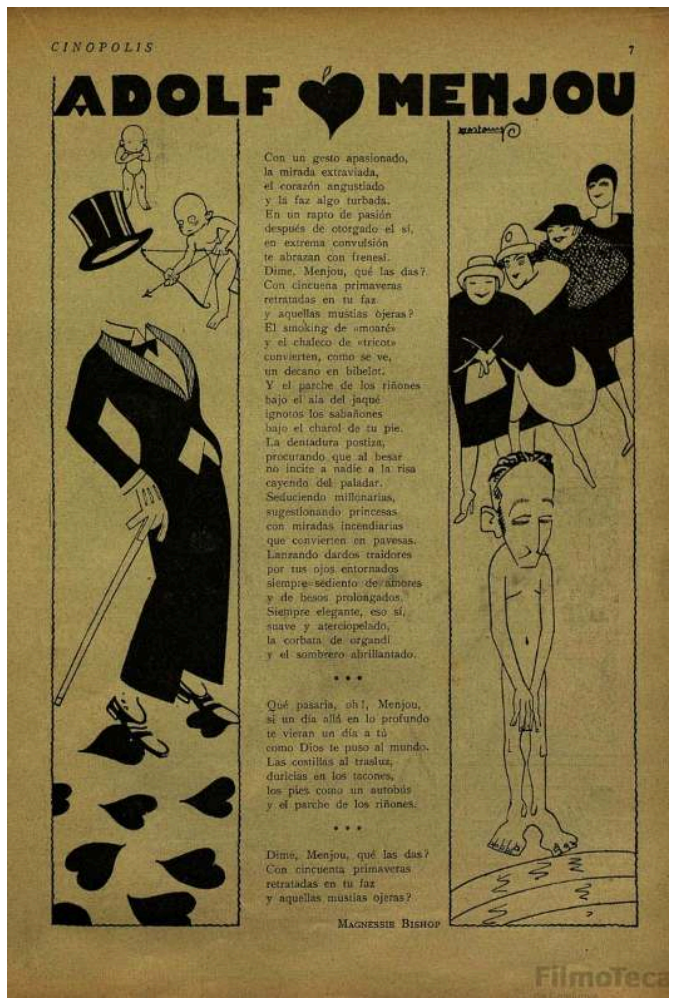


Fig. 4. Couverture du numéro 2 de Nuestro Cinema (seconde époque, février 1935).

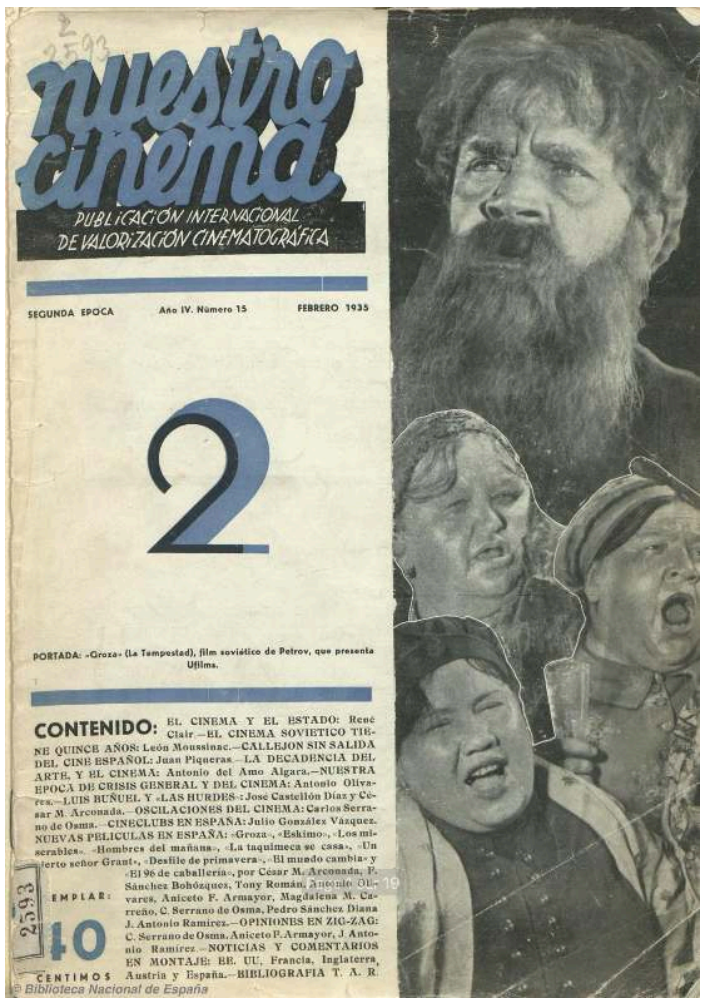

Photogramme extrait du film soviétique L'Orage (Vladimir Petrov, 1934).

Fig. 5. Couverture du numéro 10 de Nuestro Cinema (mars 1933).

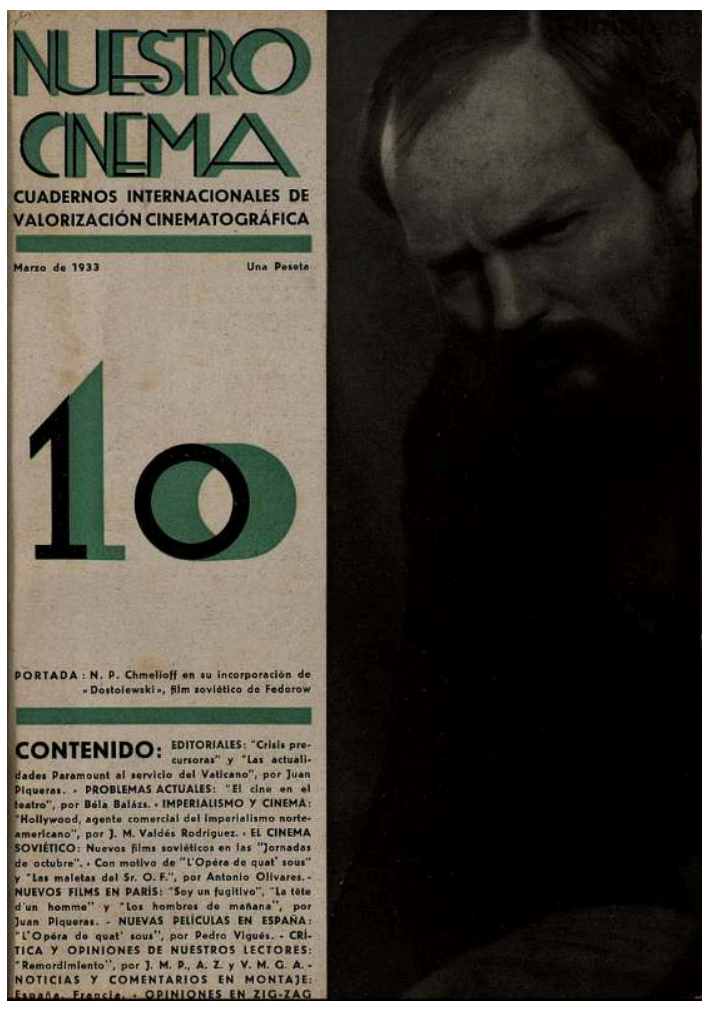

Photogramme extrait du film soviétique La Maison des morts (Vassili Fedorov, 1932). 
Le latin lover - incarné en premier lieu par Rudolph Valentino puis par de nombreux autres acteurs latins ou ayant des traits latins comme Ramón Novarro ou John Gilbert constitue l'une des principales cibles des journalistes espagnols. Les caractéristiques «féminines» - tendresse, passivité dans les relations amoureuses, élégance vestimentaire - associées à ce stéréotype expliquent son succès auprès de la gent féminine (Hansen 1991, Leconte 1996) et pouvaient à plus forte raison inquiéter des Espagnols susceptibles de l'interpréter à titre personnel, comme une remise en cause de leur propre virilité. Pour le spectateur de sexe masculin, le simple fait d'approuver ces modèles pouvait être décrypté comme le signe d'une homosexualité latente, d'autant que certains latin lovers étaient connus pour les relations homosexuelles ou bisexuelles qu'ils entretenaient dans leur vie privée. Pour cette raison, les journalistes qui n'hésitaient pas à exprimer leur fascination envers ces stars en leur consacrant des chroniques furent fortement fustigés par Piqueras et les siens. C'est le cas de Santiago Aguilar, auteur de plusieurs articles sur Valentino et autres latin lovers. Piqueras l'évoquera dans un texte où il s'oppose à Fernando Méndez-Leite, directeur de la revue Cinema :

Lui, il déteste le cinéma russe. Moi, j'admire le cinéma russe. [...] Lui, il loue ou critique en appliquant un tarif préalable, d'après ce qu'il déclare. Moi, je nie ou j'affirme librement, sans penser à cette plus-value, que je ne perçois jamais, et qui l'intéresse tant. [...] Lui, il admire Santiago Aguilar. Moi, je n'accorde aucune importance à ce Rudolph Valentino de la zarzuela, comme il se dénomme lui-même $[\ldots]^{12}$. (Piqueras 1931)

Les craintes soulevées par l'homosexualité chez les journalistes de Nuestro Cinema, voire leur aversion pour celle-ci, sont tout à fait manifestes dans la revue. Le premier numéro inclut par exemple une traduction des deux premiers volets de l'essai intitulé Réforme du cinéma (1932), de Charles Dekeukeleire, William Rombauts et Paul Werrie. Le premier aparté s'intitule «Le coiffeur et les millions» («El peluquero y los millones»). Les auteurs y citent des propos de Paul Claudel, traduits en espagnol :

Quand une jeune personne voit se dessiner à « l'écran » la physionomie attrayante de Novarro ou de Valentino et apparaitre peu à peu tous les éléments de leur regard dominateur, des pensées de toutes sortes, favorables et imprévues, s'élèvent de leur cœur troublé13. (Dekeukeleire, Rombauts et Werrie 1932)

Derrière la condamnation viscérale d'un «cinéma bourgeois » se cache la haine de l'homosexualité que ces journalistes associent aux classes sociales aisées. Depuis la fin du XIX ${ }^{e}$ siècle, l'homosexualité était en effet liée aux classes supérieures et était perçue comme " une tradition étrangère à la classe ouvrière ", un " vice des élites » (Albertini 2012), et comme une conséquence du système capitaliste qui propageait le luxe, vecteur de déchéance morale. Le régime patriarcal et homophobe de Staline présentera l'homosexualité comme un "produit de la décadence bourgeoise", une "perversion fasciste » à laquelle il opposera la " pureté morale » du prolétariat.

Rien d'étonnant, par conséquent, à ce que le cinéma russe et soviétique constitue le modèle de référence de Nuestro Cinema. Au même titre que le cinéma de Hollywood, ces productions véhiculent des stéréotypes, notamment celui du communiste au regard perçant, à la forte carrure, grand et fort, qui apparaît dans l'iconographie de la révolution russe et que la revue espagnole récupère très largement dans ses illustrations. Comme le souligne George Mosse (1997: 132), ces représentations de la masculinité forgées par le réalisme soviétique présentent par ailleurs des traits communs avec les images des nazis : « la pose inspirée, le port de tête, le regard franc et 
droit. Les idéaux de volonté de puissance, de solidité, d'énergie, n'étaient pas non plus fort différents ».

11 Parallèlement à l'exaltation d'une masculinité fondée sur la virilité et la domination, la revue laisse également entrevoir ses positions quant à la place des femmes dans la société en présentant la maternité comme une valeur fondatrice de la féminité (Fig. 6 et 7) et en fustigeant certains stéréotypes alimentés par le cinéma hollywoodien - la femme fatale, ou encore la flapper - qui incarnent la séduction et l'idée de dangerosité féminine ${ }^{14}$.

Fig. 6. Couverture du numéro 4 de Nuestro Cinema (septembre 1932).

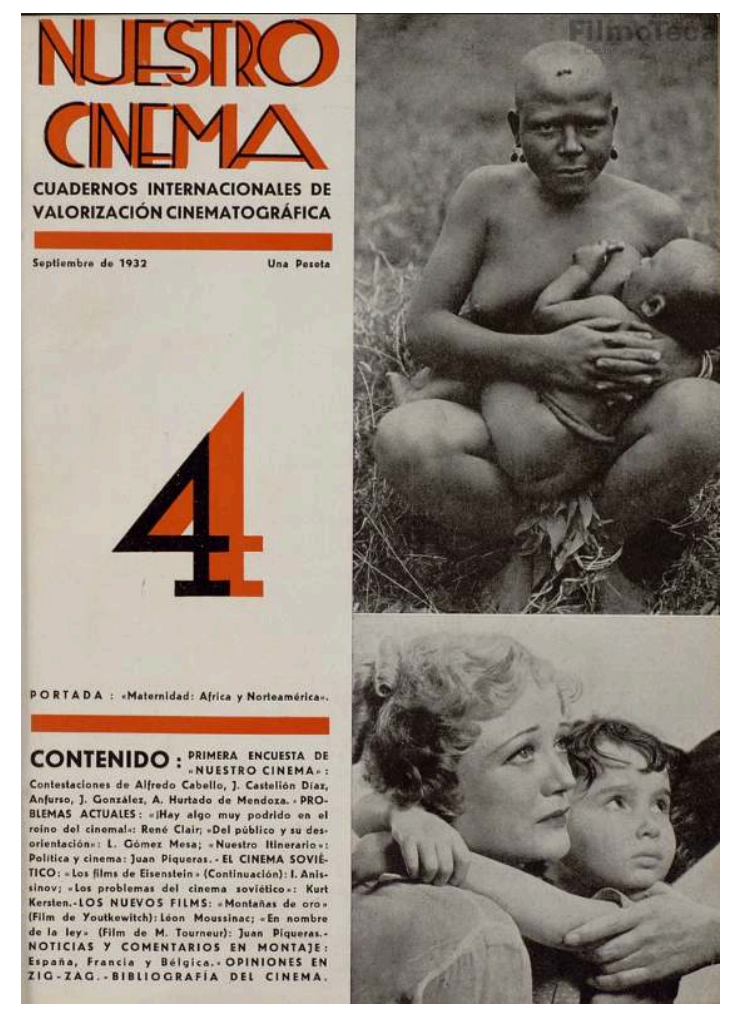

Photogrammes extraits de deux films - soviétique et hollywoodien - qui représentent la maternité 
Fig. 7. Couverture du numéro 7 de Nuestro Cinema (décembre 1932).

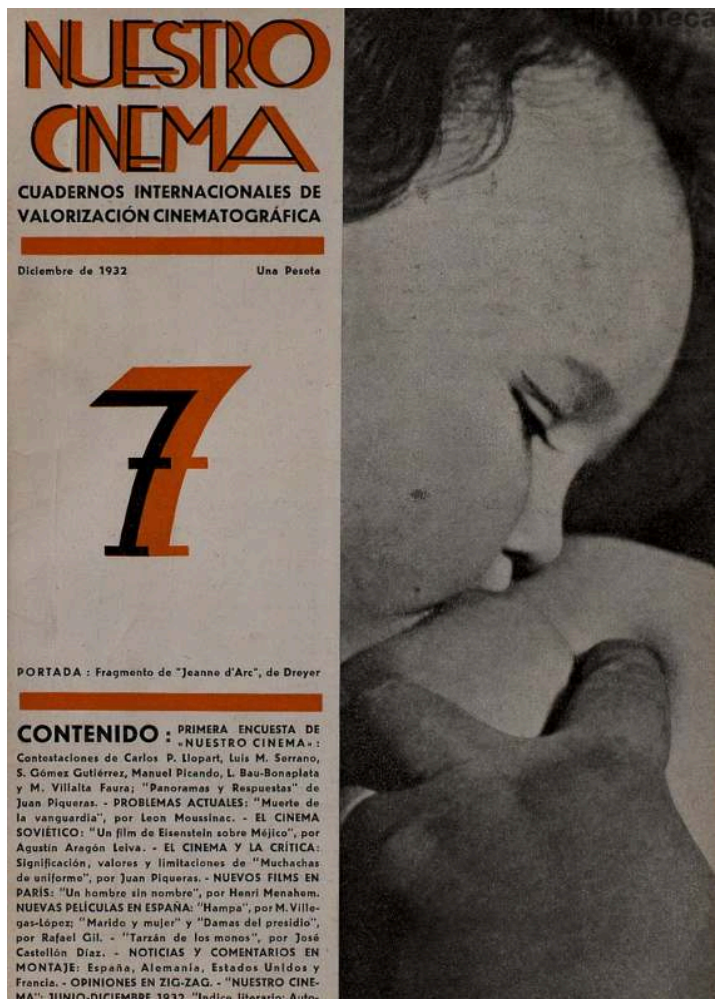

Photogramme extrait du film français La Passion de Jeanne d'Arc (Carl Theodor Dreyer, 1927).

12 L'homme féminin et la femme masculine sont souvent évoqués conjointement comme un binôme à enrayer. Dans le numéro 11, un article de Manuel Iglesias (1933) critique avec virulence «l'usine qui produit des vamps cent pour cent pur jus et de chatoyants jeunes premiers efféminés ${ }^{15}$ » et invite à " protester virilement contre la dictature de $l^{\prime}{ }^{16}{ }^{16}$. Face aux acteurs dévirilisés et aux femmes fatales qui vampent leur proie, les modèles issus du cinéma russe se révèlent extrêmement rassurants. L'absence d'individualisation des héros, de même que leur situation au sein des films - par exemple, lorsqu'ils pilotent un avion - font obstacle à la réification et au fétichisme favorisés par le grand écran et par le culte de la star (Haynes 2003 : 59). Là où la star du cinéma hollywoodien devient facilement l'objet du regard, les acteurs du cinéma russe échappent à ce voyeurisme.

\section{Le rejet de l'homme « castré »}

13 À travers la promotion du cinéma russe et soviétique - présenté comme un exemple à suivre pour le cinéma espagnol - les journalistes de Nuestro Cinema tentent de mettre à distance une culture cinématographique qu'ils assimilent explicitement à une castration. De façon non exclusive, les films de Hollywood sont les principaux pourvoyeurs de fictions cinématographiques qui mettent en scène une masculinité incomplète, tronquée, mutilée. Pour certains journalistes, le fait d'accueillir positivement les productions hollywoodiennes revient à accepter une castration métaphorique, une image qui apparaît explicitement dans les propos d'Alfredo Cabello (1932) qui, interrogé sur la situation actuelle du cinéma, répond de la façon suivante : «Sa situation? Comme tout ce qui est sous le contrôle du capitalisme : admirablement 
équipé sur le plan technique et mécanique, mais orienté à contresens. Un falsificateur de la réalité. Un trompeur. Un endormeur. Un castrateur ${ }^{17}$. » D'où la volonté d'écarter systématiquement le féminin, perçu comme dangereux et dévirilisant pour l'homme, en particulier pour l'ouvrier qui, par sa révolte, devrait incarner la force virile par excellence. C'est bien ce que suggère Castellón Díaz (1932) selon qui «le spectateur moyen souhaite voir autre chose que le baiser final de la secrétaire et du fils du patron ; ou l'épouse qui se rend à un bal masqué avec son bien-aimé, courant le risque que son mari ne découvre tout et qu'un scandale n'éclate ${ }^{18}$ ».

Un rejet viscéral des contenus sentimentaux - considérés comme dévirilisants pour l'homme qui les "consomme »- peut être constaté dans des critiques filmiques qui déplorent des situations où un personnage d'homme est représenté en position de difficulté ou d'infériorité. Un bon exemple apparaît dans le numéro 5 qui comprend une critique du film Quick (Robert Siodmak, 1932) écrite par Piqueras. Le clown Quick se trouve sous l'emprise d'une jeune femme qui fait de lui son objet, une position qui n'échappe pas au journaliste qui, pour cette raison, dénigre le film:

Sa thématique est tout à fait obsolète. Aujourd'hui, quel intérêt peut bien comporter un film qui se fonde sur l'épisode mièvre et sentimental d'un clown amoureux d'une jeune fille neurasthénique, qui ne voit en lui qu'un clown au visage peinturluré ? Autrement dit, ce n'est pas l'homme qui l'intéresse, mais le pantin qui la divertit ${ }^{19}$. (Piqueras 1932b)

Cette tendance est encore plus marquée dans le cas de films inspirés du folklore hispanique qui diffusent une certaine image de l'Espagne et de ses coutumes. Piqueras soulignera ainsi les nombreux inconvénients du film El picador (Le Picador, Lucien Jacquelux, 1932), en particulier l'apparence «efféminée » de l'acteur qui amène le journaliste à situer ce film du côté de l'espagnolade, terme qui apparaît en italique afin de rehausser son sens péjoratif et son caractère indésirable :

Nous avons récemment pu voir à Paris un film qui constitue pour ainsi dire l' espagnolade la plus fanfaronne de toutes les espagnolades produites jusqu'à ce jour. Ni les films nationaux sur l'Andalousie, ni les films français ni les films yanquees ne surpassent ce film de Jacquelux.

Nous ne souhaitons pas analyser ce film en détail car il regorge de bêtises. [...] Le protagoniste est un chanteur de l'Opéra parisien, c'est-à-dire tout ce qu'il y a de plus opposé à ce qu'exigerait le picador andalou. Le toréador est un acteur chilien (Enrique Rivero), dont l'efféminement et le manque de tempérament ont pu être largement constatés dans Le Sang d'un poète, film de Jean Cocteau. [...] Les autres interprètes sont également aux antipodes de ce que l'on pourrait espérer dans le cas d'un film de mœurs andalouses ${ }^{20}$. (Piqueras 1932c)

En revanche, les films où un personnage de sexe féminin est mis en difficulté, en particulier s'il est initialement dominateur, peuvent faire l'objet d'une critique positive. On le constate pour le film Freaks (Tod Browning, 1932), un mélodrame fantastique qui met en scène des êtres monstrueux et difformes qui se produisent dans un cirque. L'un de ces nains prénommé Hans s'éprend de Cléopâtre, la femme fatale qui, dans le but de lui dérober sa fortune, simule de faux sentiments et dit vouloir l'épouser. Elle n'hésite pas à insulter les amis de Hans et empoisonne ce dernier pour lui soutirer ses biens. Néanmoins, les nains découvrent ses manigances et préparent leur vengeance. Ce retournement rassure Piqueras qui le retranscrit avant d'émettre des commentaires laudatifs sur ce film qui, d'après lui, fait figure d'exception dans le cinéma hollywoodien :

Des silhouettes cauchemardesques apparaissent peu à peu devant la femme : le demi-homme nettoie soigneusement les canons de son pistolet; l'Homme sans 
jambes caresse son poignard gigantesque ; l'Homme-sac se faufile, le couteau entre les dents ; tous les autres, unis par le même malheur, regroupés par le même code, encerclent peu à peu la voiture de la «femme fatale», d'un air violent. Un «speaker» nous présente ensuite cette belle femme, pour laquelle des princes se sont suicidés, désormais transformée en femme-poule. Les "monstres" avaient accompli leur vengeance et avaient fait d'elle «l'un des leurs ». Ces scènes finales sont formidablement subversives. Le cinéma yanquee, où triomphent généralement la beauté et l'arrogance, ne nous a pas habitués à de telles choses ${ }^{21}$. (Piqueras 1932d)

On remarquera l'énumération d'armes à la forme phallique qui rythme le récit du journaliste et ménage le suspense. La critique de ce film témoigne d'une véritable fascination ressentie face à cette scène et d'un plaisir qui repose sur la vengeance des nains.

15 À travers ces critiques filmiques, de même que par l'exaltation du cinéma russe et d'autres cinématographies européennes qui s'inscrivent en marge des codes du cinéma hollywoodien et du star system, les journalistes de Nuestro Cinema définissent les perspectives que le cinéma espagnol devra emprunter s'il veut diffuser l'image d'une Espagne forte, où les hommes conservent un statut dominant et détiennent le monopole de la création artistique.

\section{Nuestro Cinema et la régénération de la nation}

Le rejet d'une féminité perçue comme dangereuse ne traduit pas seulement une volonté de domination des hommes sur les femmes, mais implique également une domination des hommes - les journalistes de Nuestro Cinema - sur d'autres hommes qui n'ont pas adhéré à la cause de la revue et qui continuent à alimenter le star system, manifestant leur intérêt personnel pour cette "infra-culture ». Ces derniers sont constamment rabaissés et présentés comme des hommes faibles qui se sont laissés séduire par une industrie aux effets abrutissants. Face à ces "mauvais » journalistes qui sont mis à l'écart de la masculinité hégémonique, Nuestro Cinema se présente comme la seule revue capable de remédier à la dévirilisation de la nation symbolisée par la figure de l'ouvrier qui se laisse bercer passivement par des histoires sentimentales :

Les ouvriers vont au cinéma [...] sans la moindre préoccupation, l'esprit las, endolori. [...] Et ils partagent les conflits sentimentaux des millionnaires de Palm Beach [...]. Une entreprise revêche, tenace, de déclassification, que Nuestro Cinema n'appuiera pas.

La sous-estimation à laquelle nous faisons allusion atteint en Espagne des proportions effroyables. Nous ne pouvons compter que sur la plate-forme de Nuestro Cinema, seule, isolée.

Les journaux que lisent habituellement les ouvriers espagnols [...] ne s'occupent pas du cinéma ou le conçoivent de manière indigne. Le socialiste parvient au comble de la honte: il publie chaque semaine dans ses pages les notes qu'il reçoit des distributeurs : intrigues, biographies de "stars ", la vérité sur un divorce... La Terre [...], qui s'intéresse à la [...] production lamentable de Sternberg [Blonde Vénus], commente : «Marlene... un rôle complètement différent de celui qu'elle interprète dans les films qui l'ont consacrée comme première figure du cinéma mondial... elle interprète tous les rôles, aussi difficiles soient-ils [...] $]^{22}$.» (Blanca 1933)

Les spectateurs capables de s'émerveiller face aux films de Hollywood sont eux aussi stigmatisés et féminisés en étant présentés comme de jeunes gringalets immatures, incapables de s'intéresser aux chefs-d'œuvre du cinéma russe. Comme le montrent ces 
propos de Castellón Díaz (1932), ce type de spectateur est considéré comme l'équivalent, au masculin, de la midinette :

Mademoiselle Clo n'aime que les films sentimentaux idiots de Gaynor ou l'élégance et la vigueur de ce cher Ramón Novarro. Et en ce qui concerne le jeune monsieur Adolfo, il ne faut pas lui parler d'autre chose que de Marlène et, tout au plus, de Joan : tout le reste n'est qu'idioties, rien d'autre que de solennelles idioties ${ }^{23}$.

Tout comme ses camarades, ce journaliste condamne ce qu'il dénomme «l'attitude maniérée » («el amaneramiento ») et l'« embourgeoisement " ( la emburguesación»), deux phénomènes qui semblent reliés à des conduites déviantes de la part des spectateurs et spectatrices qui imitent les stars mentionnées: «Tant que toutes les demoiselles de la péninsule ibérique rêveront d'être une Joan Crawford et tous les jeunots des Roberts Montgomerys, on ne fera rien ${ }^{24}$ » (Castellón Díaz 1932). Des craintes similaires face à l'élégance suspecte des jeunes premiers se profilent dans ces propos de J. González (1932) : « Nous devons rejeter les films américains, ce cinéma globalement stupide, qui donne à voir l'interprétation de telle ou telle star ou de tel ou tel jeunot bien apprêté ${ }^{25}$.»

Pour ces journalistes, le fait de s'exprimer dans Nuestro Cinema et de promouvoir la production russe et soviétique - ou de cinématographies européennes sans stars relève d'un véritable combat qui leur permet de retrouver une virilité mise en cause, non seulement par les modèles du star system mais aussi par les évolutions dont bénéficient les femmes dans l'Espagne de la Seconde République. Ce combat est à l'image du contenu des films qu'ils louent de façon récurrente : Quatre de l'infanterie (G. W. Pabst, 1918), Le Cuirassé Potemkine (S. M. Eisenstein, 1925), ou encore La Ligne générale (S. M. Eisenstein, 1929) figurent parmi les exemples les plus emblématiques. Ils n'hésitent d'ailleurs pas à caractériser leur démarche en termes guerriers et recourent à des métaphores qui relèvent du domaine du combat. Après avoir signalé l'égarement des masses, Arturo Casinos Guillén (1932) augure un duel sans merci : «La lutte sera acharnée. L'Europe, berceau du cinéma-art, contre la puissante Amérique et l'ignorance manifeste des masses ${ }^{26}$. » Au sein de cette guerre des nations, la production russe est présentée comme la "grande torche qui éclaire le monde" ("la magna antorcha que ilumina el mundo ») (Casinos Guillén 1932), celle qui fait triompher la virilité.

Les journalistes de Nuestro Cinema revendiquent « un cinéma substantiel, nouveau et fort » (" un cinema enjundioso[,] nuevo y fuerte ») (Piqueras 1932d), ou encore " têtu, dogmatique et violent» («terco, dogmático y violento ») (Cabello 1932). Ces adjectifs reflètent la transposition, au cinéma lui-même, de leur conception de la masculinité. On le voit dans ces quelques lignes où l'idéal d'une masculinité "forte » est appliqué au cinéma de Hollywood qui l'a incarnée à une époque révolue : «Le cinéma yanquee était un cinéma fort quand, dans son étape sportive et aventurière, il se limitait à donner une image du pays qui le produisait. Ses moments les plus personnels, ce sont ceux où il nous conduisait à l'Ouest américain ou aux stades sportifs ${ }^{27}$ » (Piqueras 1932a).

La clef de voûte de la remasculinisation de la culture cinématographique sera le réalisateur qui, dans le cinéma de Hollywood, est obscurci par la figure de la star qui tient le haut de l'affiche. Ce phénomène n'a pas lieu dans les cinématographies sans stars, dont le cinéma russe et soviétique constitue l'emblème. Les films que Nuestro Cinema cherche ainsi à promouvoir relèvent d'un "cinéma d'auteur", comme le montrent ces propos de Rafael Gil (1932) qui reflètent bien la revendication d'une cinématographie au sein de laquelle chaque film est rattaché au nom d'un auteur de sexe masculin : 
Un esprit fin, élevé, trouve dans un film de Vidor ou de Stroheim un exposant de ses sentiments, une œuvre d'art si pure qu'il peut entamer avec elle un dialogue spirituel.

Un spectateur insatisfait, qui aspire toujours à un au-delà [...] trouve dans le cinéma ce qu'il souhaite. Il peut voir un ruban de celluloïd se transformer en un drapeau qui draine derrière lui toute la masse insatisfaite lorsqu'il a été forgé par un Poudovkine ou un Eisenstein ${ }^{28}$.

Cette métaphore montre l'importance de la dimension révolutionnaire que les journalistes de Nuestro Cinema prêtent à leur démarche, et qu'ils associent à un cinéaste divinisé qui fait figure de leader. Dans cette perspective, la production russe et soviétique constitue le modèle et l'exemple à suivre, comme le souligne également Rafael Gil (1932) : « Le futur cinéma espagnol doit s'appuyer [...] sur le cinéma russe. [...] Un cinéma qui, pour y entrer, ne requiert pas un joli visage ni des muscles d'athlète, mais une culture solide, un véritable tempérament artistique ${ }^{29}$. " Une conception similaire apparaît également dans les propos d'Augusto Ysern (1932), qui pointe du doigt le contre-modèle représenté par des cinéastes espagnols :

La production espagnole doit suivre le chemin de la production russe. La Russie doit être le miroir dans lequel notre celluloïd se contemple. Toutes les tentatives cinématographiques qui ont été menées en Espagne ont donné comme fruit indiscutable un seul film : Le Village maudit ${ }^{30}$, de Florián Rey. Nous pouvons espérer bien davantage de ce dernier que d'un Perojo ou d'un Delgado ${ }^{31}$.

Ces deux réalisateurs, qui se sont inspirés du star system et qui ont tenté d'en construire un à travers leurs films, sont d'emblée situés en marge d'une élite capable de comprendre le cinéma et de l'orienter comme il se doit.

Cette étude de Nuestro Cinema vient mettre en relief un enjeu essentiel sous-tendant l'existence de cette publication qui vante sans cesse son caractère atypique et novateur par rapport à la majorité des revues de l'époque. L'équipe de rédaction - constituée exclusivement de journalistes de sexe masculin, contrairement à ce qui se produit dans les revues "hybrides" - semble en effet mener un combat destiné à restaurer une masculinité mise à mal par certaines productions filmiques et par des images de stars qui s'écartent des canons traditionnels de masculinité et de féminité. Le discours guerrier et vindicatif de ces journalistes vise à promouvoir un cinéma où l'homme conserve sa virilité et sa supériorité. En exaltant principalement le cinéma russe et soviétique, ils cherchent à redéfinir les contours d'un cinéma espagnol qui saura remettre à l'honneur cette conception d'une masculinité forte, fondée sur la force physique ainsi que sur le monopole de la «bonne » culture et de la vie intellectuelle. Leur entreprise est ainsi liée à une triple régénérescence : celle du cinéma et de la culture cinématographique, celle de la masculinité et celle de la nation, qui redeviendra forte si elle ne se laisse pas corrompre par des modèles « déviants » et indésirables. 


\section{BIBLIOGRAPHIE}

Albertini, Pierre, 2012, « Une tradition étrangère à la classe ouvrière ", 10 novembre 2012, [En ligne], http://lmsi.net/Une-tradition-etrangere-a-la, consulté le 27 novembre 2019.

Aresti Esteban, Nerea, 2010, Masculinidades en tela de juicio: hombre y género en el primer tercio del siglo XX, Madrid, Cátedra.

Baecque, Antoine de, 2003, La Cinéphilie. Invention d'un regard, histoire d'une culture (1944-1968), Paris, Fayard.

Connell, Robert William et Messerschmidt, James W., 2015, « Faut-il repenser le concept de masculinité hégémonique ? Traduction coordonnée par Élodie Béthoux et Caroline Vincensini », Terrains \& travaux, $n^{\circ} 27$, p. 151-192, [En ligne], https://www.cairn.info/revue-terrains-ettravaux-2015-2-page-151.htm, consulté le 27 novembre 2019.

DOI : $10.3917 / \mathrm{tt} .027 .0151$

Coquillat, Michelle, 1982, La Poétique du mâle, Paris, Gallimard.

Farges, Patrick, 2012, « Masculinités et masculinisme ? (1880-1920) », Les carnets de recherche du CIERA, [En ligne], http://ciera.hypotheses.org/322, consulté le 27 novembre 2019.

Hansen, Miriam, 1991, « Pleasure, Ambivalence, Identification: Valentino and Female Spectatorship », dans C. Gledhill (dir.), Stardom: Industry of Desire, Londres, Routledge, p. 259-282.

Haynes, John, 2003, New Soviet Man. Gender and Masculinity in Stalinist Soviet Cinema, Manchester, New York, Manchester University Press.

Leconte, Loredana, 1996, « Latin Charm. Inventing Rudolph Valentino », dans G. Malossi (dir.), Latin Lover: The Passionate South, Milan, Charta, p. 81-93.

Llopis, Juan Manuel, 1988, Juan Piqueras, el Delluc español, Valence, Filmoteca Generalitat Valenciana, 2 vol.

Monterde, José Enrique, 2011, « Crítica », dans C. F. Heredero, E. Rodríguez Merchán et I. Giroud (dir.), Diccionario del cine iberoamericano: España, Portugal y América, Madrid, Sociedad General de Autores y Editores, Fundación Autor.

Mosse, George Lachmann, 1997, L'Image de l'homme. L'invention de la virilité moderne, Paris, Abbeville.

Sellier, Geneviève, 2005, La Nouvelle Vague : un cinéma au masculin singulier, Paris, CNRS Éditions.

\section{Corpus}

«Aurelio », 1922, « Estrellas y luceros del firmamento cinematográfico », Cine Popular, 8 mars $1922, \mathrm{n}^{\circ} 54$

« Magnessie Bishop », 1929, « Adolfo Menjou », Cinòpolis, 26 janvier 1929, nº 1.

Blanca, Antonio, 1933, « Problemas actuales: subvaloración del cinema », Nuestro Cinema, avrilmai $1933, \mathrm{n}^{\circ} 11$.

Buñuel, Luis, 1928, « Variaciones sobre el bigote de Menjou », La Gaceta Literaria, juin 1928, n 35. 
Cabello, Alfredo, 1932, « Primera encuesta de Nuestro Cinema. Respuesta de Alfredo Cabello », Nuestro Cinema, septembre $1932, \mathrm{n}^{\circ} 4$.

Casinos Guillén, Arturo, 1932, « Primera encuesta de Nuestro Cinema. Respuesta de A. Casinos Guillén », Nuestro Cinema, novembre 1932, nº 6.

Castellón Díaz, José, 1932, « Dos argumentos », Nuestro Cinema, novembre 1932, nº 6.

Dekeukeleire, Charles, Rombauts, William et Werrie, Paul, 1932, « Reforma del Cinema », Nuestro Cinema, juin 1932, $\mathrm{n}^{\circ}$ 1, juin 1932.

Gil, Rafael, 1932, « Primera encuesta de Nuestro Cinema. Respuesta de Rafael Gil », Nuestro Cinema, août $1932, \mathrm{n}^{\circ} 3$.

González, J., 1932, « Primera encuesta de Nuestro Cinema. Respuesta de J. González », Nuestro Cinema, septembre 1932, $\mathrm{n}^{\circ} 4$.

Iglesias, Manuel, 1933, «El caso Scottsboro », Nuestro Cinema, avril-mai 1933, nº 11.

Piqueras, Juan, 1931, « Revisión de gente y gentecilla cinematográfica: Fernando Méndez-Leite, director de CINEMA », Popular Film, 19 novembre 1931, nº 275.

Piqueras, Juan, 1932a, « Nuestro itinerario: política y cinema », Nuestro Cinema, septembre 1932, $\mathrm{n}^{\circ} 4$.

Piqueras, Juan, 1932b, « Los nuevos films: Quick », Nuestro Cinema, octobre 1932, nº 5.

Piqueras, Juan, 1932c, « El Picador: film francés de Jacquelux », Nuestro Cinema, octobre 1932, nº 5.

Piqueras, Juan, 1932d, « Barnum (Freaks): film yanqui de Tod Browning », Nuestro Cinema, novembre $1932, \mathrm{n}^{\circ} 6$.

Piqueras, Juan, 1933, « Editoriales: crisis precursoras », Nuestro Cinema, mars 1933, nº 10.

Piqueras, Juan, 1935, « Callejón sin salida del cine español », Nuestro Cinema, février 1935, nº 2.

Ysern, Augusto, 1932, " Primera encuesta de Nuestro Cinema. Respuesta de Augusto Ysern ", Nuestro Cinema, août 1932, $\mathrm{n}^{\circ} 3$.

\section{NOTES}

1. En Espagne, ce paradigme s'impose dès le début du $x^{e}$ siècle avec les deux «doyennes» de la presse cinématographique espagnole, Arte y cinematografía (1910-1936) et El Cine (1912-1935). La presse spécialisée connaîtra son essor à partir de la seconde moitié des années 1920 suite à l'apparition d'autres publications arborant des contenus plus riches et élaborés ainsi qu'une meilleure qualité d'impression. Ce modèle de presse populaire et "hybride » sera progressivement remplacé, dans les années 1950-1960, par le modèle dominant de la revue d'art et d'essai, plus sélective et élitiste.

2. Militant du Parti communiste, Piqueras vivait depuis 1930 à Paris, où la revue était éditée avant d'être imprimée à Barcelone. Nuestro Cinema totalise dix-sept numéros, publiés en deux «époques»: juin 1932-octobre 1933 (13 numéros) puis janvieraoût 1935 (4 numéros).

3. C'est aussi le point de vue qui a pu être émis par des chercheurs et historiens du cinéma. José Enrique Monterde (2011:24) affirme par exemple qu'il s'agit de «la revue 
de critique cinématographique la plus significative et la plus profonde qui a été éditée en Espagne jusqu'à nos jours» («la más significativa y profunda revista de crítica cinematográfica editada en España hasta el momento »). De même que pour toutes les citations en espagnol, nous traduisons.

4. « En este preciso instante, al hablar de cinema hispánico, debemos circunscribirnos a su ángulo industrial, puesto que no podemos conducirle a otro. »

5. «La prensa cinematográfica, en general, es de lo más rastrero y putrefacto que se publica actualmente. »

6. L'orientation de la production nationale constitue en effet l'objectif constant de la revue qui transmettra des positions opposées à celles d'autres publications qui tentent pour leur part de fomenter un star system national. L'enquête lancée par Nuestro Cinema à partir de son numéro 3 - et dont nous citerons des extraits tout au long de cet article - a pour objectif d'influencer d'une manière bien précise l'évolution et les caractéristiques des films espagnols. Ce sondage invite des intellectuels à s'exprimer à ce sujet en répondant à différentes questions : "Que pensez-vous du cinéma et de sa situation actuelle?», "Quel genre cinématographique doit-on cultiver en particulier?» (« ¿Qué piensa del cinema y de su posición actual?», «¿Qué género cinematográfico cree que debe cultivarse más atentamente?»), etc.

7. Pour certains, cette adhésion sera temporaire et restera une expérience de jeunesse : les cinéastes Carlos Serrano de Osma, Antonio Del Amo et Rafael Gil n'hésiteront pas à mettre leurs films au service des idéaux conservateurs du franquisme. Il convient donc d'interpréter cet engagement politique comme un combat qui, pour ces intellectuels, représente l'occasion de s'investir dans une cause qui leur donne le sentiment de s'affirmer en tant qu'hommes à travers une lutte politique qui revêt des accents guerriers et révolutionnaires.

8. Substantif masculin qui peut désigner une étoile ou un astre, mais qui est aussi utilisé pour renvoyer à la planète Vénus, une possibilité qui ne semble pas incommoder l'auteur malgré les rapprochements possibles avec la déesse du même nom. Précisons néanmoins que le substantif espagnol « planeta » est de genre masculin, ce qui empêche sans doute cette assimilation.

9. «Bien está que los ingleses no distingan de sexo en los seres inanimados y cosas del otro mundo, pero en España somos más afortunados y hacemos una sabia distinción y hasta separación de sexos en la gramática. [...]. Nosotros protestamos de que Charles Chaplin sea "estrella" y lo sea también el fantástico e inquieto Douglas Fairbanks [...]. Creemos que sería más caritativo darles el feliz apelativo de "luceros", haciendo una sabia diferencia entre "ellas" y "ellos", quitando a ellos esas faldas gramaticales que la irreligiosidad e iconoclastia de unos cuantos herejes del verbo les han colocado para su ridículo. Llamémosles luceros, todo lo refulgentes que su vanidad y valía exijan, pero dejemos para ellas el dulcemente femenino calificativo de "estrella", tan justificante y amable y galanteadora prueba de agradecimiento hacia esas bellas y simpáticas mujeres que tan gratos instantes nos hacen pasar con sus grandes creaciones. »

10. Le titre complet de cette publication éditée à Barcelone en 1929 - Cinòpolis: la revista humorística del cinema- indique bien la prise en compte de la culture cinématographique sous un angle comique et satirique.

11. «El smoking de moaré / y el chaleco de "tricot" / convierten, como se ve, / un decano en bibelot.» 
12. «Él es enemigo del cine ruso. Yo soy admirador del cine ruso. [...] Él elogia o critica aplicando una previa tarifa, según declara. Yo niego o afirmo libremente, sin pensar en ese tanto por ciento, que no recibo nunca, y en el que él tanto se fija. [...]. Él es admirador de Santiago Aguilar. Yo no le concedo importancia alguna a este Rodolfo Valentino de la zarzuela, como él mismo se llama. »

13. "Cuando una persona joven ve dibujarse sobre el "écran" la fisonomía atrayente de Novarro o de Valentino y reunirse poco a poco todos los elementos de su mirada dominadora, toda suerte de pensamientos, favorables e imprevistos, se elevan de su corazón turbado."

14. À cet égard, précisons que, malgré son orientation anti-star, Nuestro Cinema n'hésitera pas à inclure, de temps à autre, la photographie d'une actrice sensuelle, cultivant une forme d'érotomanie qu'Antoine de Baecque a également décelée chez les cinéphiles de la Nouvelle Vague française (De Baecque 2003:268) et qui traduit une considération de la femme en tant que simple objet érotique (Sellier 2005 : 26-31).

15. «La actual fábrica de vampiresas cien por cien y de vistosos galanes afeminados ».

16. «Protestar virilmente contra la dictadura del oro ».

17. « ¿Su situación? Como todo lo regido por alta mano capitalista, dotado de un admirable instrumental técnico-mecánico, pero orientado a contracorriente. Falseando la realidad. Engañando. Adormeciendo. Castrando.»

18. «El espectador medio desea ver otra cosa que el beso final de la mecanógrafa y el hijo del patrón; o que la esposa se vaya a un baile de máscaras con su querido, expuesta, claro es, a que el marido lo descubra todo y se arme un escándalo. »

19. « Su asunto es una cosa vieja y trasnochada. Hoy no puede interesar a nadie una película cuya base se apuntala sobre el episodio sensiblero y sentimental de un payaso enamorado de una muchacha neurasténica, que solamente ve en él al payaso con cara embadurnada. Es decir, que no es el hombre quien le interesa, sino el muñeco que la entretiene.»

20. «En París termina de presentarse un film que viene a ser algo así como la españolada más españolada de todas las españoladas hechas hasta la fecha. Ni los films nacionales sobre Andalucía, ni los franceses, ni los yanquis son peores que esta película de Jaquelux. No queremos analizar el film detenidamente porque está lleno de disparates. [...] Su protagonista es un cantante de la Opera parisiense, que es tanto como decir lo más opuesto a lo que exigiría el picador andaluz. El torero es un actor chileno (Enrique Rivero), cuyo afeminamiento y falta de temperamento ha quedado bien patente en La sangre de un poeta, film de Jean Cocteau [...]. Los demás intérpretes son también gentes opuestas a las que pudiesen desearse para un film de costumbres andaluzas. »

21. «Ante la mujer comienzan a aparecer figuras de pesadilla: el Medio hombre limpia cuidadosamente los cañones de su pistola; el Hombre sin piernas acaricia su navaja gigantesca; el Hombre saco se arrastra con su cuchillo entre los dientes; todos los demás, unidos por la misma desgracia, agrupados por igual contraseña, van rodeando el coche de la "mujer fatal" en actitudes violentas. Un "speaker" nos presenta después a esta mujer bella, por la que se suicidaron príncipes auténticos, transformada en la Mujer gallina. Los "monstruos" habían cumplido su venganza y habían hecho de ella "uno de los suyos". Estas escenas finales son de una subversión formidable. Es algo a lo 
que no nos tiene acostumbrado ese cinema yanqui en el que siempre triunfa lo bello y lo arrogante. »

22. « Los obreros acuden al cinema [...] con el ánimo laxo, cansado, dolorido. [...] Y comparten los conflictos sentimentales de los millonarios en Palm Beach [...]. Una labor honda, tenaz, de desclasificación, que no hay que descubrir en Nuestro Cinema. [...] La subvaloración a la que aludimos alcanza en España proporciones aterradoras. Sólo contamos con la plataforma de Nuestro Cinema, única, aislada. Los diarios que suelen leer los obreros españoles [...] no se ocupan del cinema o lo conciben indignamente. El Socialista llega al máximo ludibrio: publica en sus planas semanales todas las notas que recibe de las distribuidoras: argumentos, biografía de "estrellas", la verdad sobre un divorcio... En La Tierra [...], ocupándose de la misma y lamentable producción de Sternberg [La Venus rubia]: "Marlene... un papel completamente distinto al interpretado en las cintas que la consagraron como primerísima figura del cinema mundial... abarca todos los papeles por difíciles que sean [...]”.»

23. «A la señorita Clo sólo le gustan los films sentimentales idiotas de la Gaynor o la elegancia y vigor de Ramoncito Novarro. Y, por su parte, al señorito Adolfo que no se le hable más que de Marlene, y todo lo más de Joan: las demás cosas son idioteces, nada más que unas solemnes idioteces. »

24. «Mientras que cada una de las señoritas ibéricas sueñe con ser una Joan Crawford y todos los pollos unos Roberts Montgomerys, no se hará nada. »

25. « Hemos de descartar los films americanos, ese cinema en su generalidad estúpido, que proporciona la actuación de determinada estrella o apuesto jovenzuelo. »

26. «La lucha, pues, es enconada. Europa, cuna del cine-arte, batalla, en duelo a muerte, contra la potente América y la manifiesta ignorancia de las masas. »

27. «El cine yanqui era un cine fuerte cuando, en su etapa deportiva y aventurera, se limitaba a manifestarse en igual forma en que vivía el país que le daba. Sus momentos más personales, son aquellos en que nos llevaba al Oeste o a los estadios deportivos. "

28. «Un espíritu selecto, elevado, encuentra en un film de Vidor o de Stroheim un exponente de sus sentimientos, una obra de arte tan puro que puede entablar con ella un diálogo espiritual. Un espectador insatisfecho, que ansía siempre un más allá [...] encuentra en el cinema lo que quiere. Ve un rollo de celuloide que se convierte en una bandera que arrastra tras de sí a toda la masa insatisfecha cuando ha sido forjado por un Pudovkin o un Eisenstein. "

29. «El futuro cinema español debe basarse [...] en el cine ruso. [...] Un cinema que, para entrar en él, no sea necesario un rostro bonito ni unos músculos de atleta, sino una cultura cuidada, un verdadero temperamento artístico. »

30. Ce film dépeint la vie quotidienne de la Castille rurale des années 1920, marquée par l'immobilisme et la perpétuation des conceptions les plus rétrogrades. La trajectoire d'Acacia, l'héroïne interprétée par Carmen Viance, permet de représenter la position de rédempteur qu'occupe l'homme dans cette société fortement patriarcale où la femme reste cantonnée aux tâches domestiques et au devoir d'aller à l'église. Ayant abandonné son village pour la ville afin de fuir la famine, Acacia est répudiée par ses proches. Son mari la retrouve plus tard dans un bar et l'on comprend qu'elle est tombée dans la prostitution. Malgré cela, il décide de la rapatrier au foyer mais lui interdit tout contact avec leur fils. Elle sombre dans la misère et la folie avant d'obtenir finalement le pardon de son époux. Florián Rey réalisera une version sonorisée de ce film en 1942, 
ce qui montre la compatibilité entre les valeurs contenues dans sa trame et les principes de la dictature franquiste.

31. «La producción española debe ser encauzada hacia la rusa. Rusia ha de ser el espejo en que se mire nuestro celuloide. Todos los ensayos de cine que se han hecho en España han dado como fruto indiscutible un solo film: La aldea maldita de Florián Rey. De él podemos esperar mucho más que de un Perojo o de un Delgado. »

\section{RÉSUMÉS}

Cet article étudie la conception de la masculinité revendiquée par Nuestro Cinema, une revue de cinématographie qui, dès son premier numéro, s'auto-désigne comme la «meilleure » publication spécialisée, dépourvue de contenus frivoles et sentimentaux par opposition à la plupart des autres revues. Affiliée au Parti communiste, Nuestro Cinema transmet paradoxalement une conception élitiste de la culture cinématographique qui révèle une véritable crise identitaire chez des Espagnols soucieux de contrecarrer le pouvoir que les femmes pourraient conquérir grâce au cinéma, d'autant plus qu'au début des années 1930 le statut des Espagnoles connaît des évolutions significatives en Espagne. La défense d'un système patriarcal ainsi que d'une masculinité hégémonique et normative se fonde notamment sur la promotion du cinéma russe et soviétique qui présente des modèles bien différents de ceux du cinéma hollywoodien. L'analyse des articles et des critiques filmiques permet de rendre compte d'une volonté de régénération de la masculinité et de la nation qui doit s'appuyer sur le cinéma, conçu et orienté dans un sens bien spécifique.

This paper analyzes the conception of masculinity claimed by Nuestro Cinema, a film journal which, from its first issue, calls itself the «best » specialized publication, free from frivolous and sentimental contents in contrast to most other journals. Affiliated with the Communist Party, Nuestro Cinema paradoxically transmits an elitist conception of cinematographic culture which reveals a real identity crisis among Spanish men who are trying to counteract the power that women could reach through cinema, all the more as women's status is experiencing an important evolution in Spain in the early 1930s. The protection of patriarchy and of a hegemonic and normative masculinity goes along with the promotion of the Russian and Soviet cinema whose models are far different from those of Hollywood cinema. The study of articles and film reviews helps to throw light on the will to regenerate masculinity and nation, a process which must be based on cinema, considered and directed in a specific way.

\section{INDEX}

Mots-clés : Espagne, années 1930, presse cinématographique, cinéphilie, patriarcat, star system, cinéma

Keywords : Spain, 1930s, film press, cinephilia, patriarchy, star system, cinema 
AUTEUR

ÉVELYNE COUTEL

ENS de Lyon, IHRIM (UMR 5317) 